\title{
Attentional biases toward emotional images in the different episodes of bipolar disorder: An eye-tracking study
}

\author{
Ana García-Blanco ${ }^{\mathrm{a}, \mathrm{b}, *}$, Ladislao Salmerón $^{\mathrm{a}}$, Manuel Perea ${ }^{\mathrm{a}}$, Lorenzo Livianos ${ }^{\mathrm{b}}$ \\ a ERI-Lectura, Universitat de València, Valencia, Spain \\ ' Servicio de Psiquiatría, Hospital Universitari i Politècnic "La Fe", Valencia, Spain
}

\section{A R T I C L E I N F O}

\section{Article history:}

Received 21 April 2013

Received in revised form

24 December 2013

Accepted 26 December 2013

Available online 3 January 2014

Keywords:

Bipolar disorder

Cognitive biases

Selective attention

Eye tracking

\begin{abstract}
A B S T R A C T
Attentional biases toward emotional information may represent vulnerability and maintenance factors in bipolar disorder (BD). The present experimental study examined the processing of emotional information in $\mathrm{BD}$ patients using the eye-tracking technology. Bipolar patients in their different states (euthymia, mania, depression) simultaneously viewed four pictures with different emotional valence (happy, neutral, sad, threatening) for $20 \mathrm{~s}$ while their eye movements were monitored. A group of healthy individuals served as the control. The data revealed the following: (i) a decrease in attention to happy images in BD patients in their depressive episodes compared to healthy individuals, and (ii) an increase in attention to threatening images in BD patients (regardless of their episode) relative to the healthy controls. These biases appeared in the late stages of information processing and were sustained over the $20 \mathrm{~s}$ interval. Thus, the present findings reveal that attentional biases toward emotional information can be a key feature of $\mathrm{BD}$, in that: (i) an anhedonic lack of sensitivity to positive stimuli during the bipolar depressive episode may be considered a maintaining factor of this clinical state, and (ii) the trait-bias toward threat, even in asymptomatic patients, may reflect a marker of vulnerability in BD.
\end{abstract}

(c) 2014 Elsevier Ireland Ltd. All rights reserved.

\section{Introduction}

Individuals with bipolar disorder (BD) present with succeeding episodes of mania, depression, and euthymia (i.e., no symptoms), which involve important fluctuations in mood, cognitive functioning, and social behavior (see Mansell et al., 2007, for a review). Understanding the underlying affective/cognitive mechanisms involved in these episodes may prove to be helpful in the diagnosis and treatment of BD. A very useful strategy to study these mechanisms in detail is to assess variations in selective attention toward emotional stimuli during the different episodes of BD (e.g., see GarcíaBlanco et al., 2013, for recent evidence with emotional words).

At the theoretical level, cognitive models of BD (e.g., Beck, 1976) postulate the existence of positive schemata in mania and negative schemata in depression that relate to unrealistic attitudes about oneself, the world, and the future. In this framework, how information is attended to, interpreted, and remembered by $\mathrm{BD}$ individuals depends on their congruency with the mood state (depressed patients prefer negative information, while manic patients prefer positive information). These cognitive biases may constitute a factor of emotional vulnerability related to the disorder in terms of whether they

\footnotetext{
* Corresponding author at: ERI-Lectura, Universitat de València, Av. Blasco Ibáñez, 21, 46010 Valencia, Spain. Tel.: +34 963 864547; fax: + 34963864697.

E-mail address: ana.garcia-blanco@uv.es (A. García-Blanco).
}

exhibit some degree of stability independent of the symptomatology (i.e., the biases remain even during euthymic states; see Alloy et al., 2006, for review). Furthermore, the persistence of mood-congruent attentional biases along acute episodes (mania and depression) could hinder emotional self-regulation and contribute to the maintenance of affective symptomatology.

At the empirical level, only a few studies have examined these biases in individuals with BD (e.g., Chen et al., 2006; Jongen et al., 2007; Wessa et al., 2007; García-Blanco et al., 2013). Wessa et al. (2007) employed an Affective Go/No-go Task with an event-related fMRI paradigm in euthymic BD patients and healthy individuals during the processing of threatening, happy, and neutral faces. Participants were required to detect relevant targets (e.g., "happy" faces) and inhibit their responses to competing stimuli, which were presented for relatively long exposure durations (permitting awareness of the stimuli). Although the researchers failed to find differences between asymptomatic BD patients and healthy individuals in the behavioral responses, the fMRI data revealed abnormal activation during the processing of happy and threatening faces in the patients. GarcíaBlanco et al. (2013) compared BD patients in their manic, depressive, and euthymic states during the processing of "happy" and "sad" words in an Affective Go/No-go Task. They found that patients detected "happy" words more rapidly in their manic episodes, whereas in their depressive episodes, they more quickly detected "sad" words. Note that the euthymic patients and healthy individuals produced similar response times with "happy" and "sad" words. 
Other studies examined whether mood-congruent biases occur either in earlier states of information processing, that is, when emotional words were presented briefly (Jongen et al., 2007), or prior to awareness, that is, when attention was directed toward a non-emotional cue (i.e., naming the color of facial expressions; Chen et al., 2006). Jongen et al. (2007) employed a modified Dot Probe Task in a Spatial Cueing Paradigm with bipolar patients (in their depressive and euthymic states) and healthy controls. Participants were presented with a pair of words (one neutral and the other emotional [positive or negative]) for $800 \mathrm{~ms}$. Then, a probe appeared in the localization of one of the words. Participants were asked to press where the probe was presented as quickly as possible. Jongen et al. (2007) found longer response times in the depressed group than in the control group when an emotional word preceded the probe (regardless of the valence); however, the parallel difference between the euthymic group and the healthy controls was not significant.

In an fMRI study involving emotional recognition, Chen et al. (2006) presented facial expressions (neutral, sad, threatening, or happy) depicted with different colors to bipolar patients (in their depressive and manic phases) and healthy individuals. The participants responded to the facial expressions (explicit recognition) or to the colors (implicit recognition), depending on the experimental block. Implicit recognition generated a general emotional effect that was similar across the groups. More importantly, in the explicit recognition task, both depressed and manic patients showed abnormal neural responses to sad, happy, and threatening faces, compared to the healthy controls. Taken together, at the behavioral level, when the emotional information is processed consciously, attentional biases are only present in symptomatic BD patients (García-Blanco et al., 2013). However, in fMRI studies, abnormal brain activation was found during the processing of threat in both asymptomatic (Chen et al., 2006) and symptomatic patients (Wessa et al., 2007). When emotional information is processed by symptomatic patients prior to awareness (see Chen et al., 2006) or in earlier states (Jongen et al., 2007), there is a general emotional bias, without a distinction in terms of moodcongruent information.

Although the tasks employed in the above-cited experiments offer valuable information about underlying affective/cognitive processes, there is an obvious limitation. Response time (task) only offers one data time at the end of the information processing: similarly, fMRI presents very limited temporal resolution. To track the time course of attentional biases in the different BD episodes, we recorded participants' eye movements. Eye movements are related to cognitive processes during visual tasks, and shifts in gaze position closely follow-and are guided by-shifts in attentional focus (see Rayner, 2009, for review). Measuring eye movement avoids the problems of slow responses and the motor responses observed in mental illness. Thus, eye-tracking methodology is ideally suited for measuring selective attention to complex visual stimuli that are presented simultaneously and compete for the observer's attention, thereby creating a strong test for information processing bias (Hermans et al., 1999). Additionally, the recording of the participants' eye movements is useful to assess attentional biases across relatively long periods of time, in that participants can scan and re-scan the different (emotional) images.

Only a few published studies have monitored participants' eye movements during sustained attention in patients with mood disorders, and this always been restricted to major depressive disorder (MDD; see Armstrong and Olatunji, 2012, for a metaanalytical review). Of particular relevance here is the free-viewing task, in which participants are required to focus on a central fixation point, and then asked to scan/re-scan several stimuli (2-4 stimuli, e.g., images, faces, or words) presented simultaneously, depicting differing valences (happy, sad, threat or neutral) for around $10-30 \mathrm{~s}$. A number of studies assessed the maintenance of attention over the entire trial (e.g., by measuring the total fixation time on each image; see Eizenman et al., 2003; Kellough et al., 2008; Leyman et al., 2011; Sears et al., 2010, 2011; Ellis et al., 2011). Depressed individuals attended less to happy stimuli than nondepressed individuals (i.e., an "anhedonic bias" was found; Kellough et al., 2008; Leyman et al., 2011; Sears et al., 2010, 2011; Ellis et al., 2011).

Two of the above-cited studies also found that depressed individuals paid more attention to sad stimuli relative to healthy controls (Eizenman et al., 2003; Kellough et al., 2008). However, no study found differences in the eye-gaze patterns related to threatening stimuli between depressed and non-depressed individuals (Armstrong and Olatunji, 2012). To determine the presence of attentional biases in the earliest stages of information processing, several studies examined the location of first fixation on a particular image (Kellough et al., 2008; Sears et al., 2010, 2011). Moreover, albeit not conclusively, individuals with MDD tended to orient their initial fixation to happy stimuli less frequently than healthy controls (Armstrong and Olatunji, 2012).

Given that previous eye-tracking experiments suggested moodcongruent biases related to emotional information in MDD-which was particularly strong in the late stages of information processing (i.e., during the maintenance of attention over $5 \mathrm{~s}$ segments)-one important research question is whether these biases also apply to BD patients (see Beck, 1976, for a theoretical model). Of particular relevance here is that recent theoretical proposals have emphasized the distinctive features of BD relative to MDD (Mansell et al., 2007). We must keep in mind the following in terms of attentional preferences: (i) bipolar depression usually manifests psychotic and melancholic symptoms; (ii) manic patients show conflicting positive and negative appraisals; and (iii) manic patients are characterized by impulsivity and vigilance deficits that can affect the time course of attentional preferences.

To examine the emotional modulation of attentional processing in individuals along the three episodes of $\mathrm{BD}$, we used a similar paradigm to that employed by Kellough et al. (2008). Participants were simultaneously presented with four images (neutral, dysphoric, happy, and threatening). To explore whether or not attentional biases (assessed by total fixation time on pictures) were sustained over time, the exposure time for each trial was divided into $5 \mathrm{~s}$ segments. To assess whether orientation biases existed in the earliest stages of processing, we also examined the location of the first fixation. Unlike in Kellough et al. (2008), each trial was displayed for 20 instead of $30 \mathrm{~s}$ to adapt the task to the characteristics of our sample. Pilot testing showed that symptomatic patients had difficulty completing longer versions of the experiment.

We had a number of specific goals in accordance with previous (behavioral) findings in BD and eye-tracking evidence in MDD. First, we examined whether attentional mood-congruent biases are present in manic and depressive episodes, that is, whether the individuals in the manic group attended more to happy images than the healthy controls, and whether the individuals in the depressed group attended more to sad images than the healthy controls in terms of fixation time and/or frequency (see García-Blanco et al., 2013, for behavioral evidence with emotional words). Second, we examined whether previously reported abnormal brain activation during threat processing is reflected in the abnormal scanning of threatening pictures in BD patients, regardless of their mood episodes, relative to the healthy controls (i.e., whether abnormal attentional processing toward threatening stimuli is an inherent trait of BD; see Chen et al., 2006; Wessa et al., 2007, for fMRI evidence). Third, we examined whether attentional biases are sustained over short periods of time (i.e., whether there were differences across time segments in 
attentional preferences), as occurs in MDD (see Kellough et al., 2008). Finally, we examined whether attentional biases are present in attentional orientation (i.e., whether participants directed their first fixation toward particular emotional images depending on their episode; see Armstrong and Olatunji, 2012, for evidence with MDD individuals).

\section{Method}

\subsection{Participants}

Sixty-six patients with BD who were in distinct mood states-depressive $(n=20)$, euthymic $(n=23)$, and manic $(n=23)$-took part in the experiment. Two patients experiencing manic episodes refused to participate. The patients were recruited from in-patient wards $(n=41)$ and from the Bipolar Disorders Unit for outpatients $(n=25)$ in the Psychiatry Department at the Hospital Universitario y Politécnico La Fe (Valencia, Spain). An additional control group of 20 healthy individuals was recruited by advertising in the community.

This study was authorized by the ethics committee of the Health Research Institute "La Fe", and all participants provided written informed consent before inclusion in the study. Participants were excluded depending on the following criteria: neurological history, major medical disorders likely to affect cognition, and difficulty in obtaining stable eye tracking (e.g., interference from glasses, frequent crying, or eye diseases). Additional exclusionary criteria for the patients were as follows: use of non-psychotropic medication which could influence cognition (e.g., treatment with corticoids); other psychiatric diagnoses based on DSM-IV criteria (American Psychiatric Association [APA], 2000); and having undergone electroconvulsive therapy in the previous 3 months. Healthy controls were excluded if there was any psychiatric history or use of medications that might considerably affect cognition.

Patients fulfilled the DSM-IV criteria for BD, and were included in the manic, depressed, or euthymic groups at the time of assessment. Psychiatrists in the department referred appropriate patients for this study. A clinical interview and case note review were used for establishing the diagnoses. Every patient had to present at least one manic episode. DSM-IV diagnoses were corroborated by the responsible psychiatrist and by a postgraduate clinical psychology intern. In order to warrant the exclusion of mixed states, as well as the absence of affective symptoms in euthymic patients and healthy participants, two clinical scales were applied, specifically the Beck Depression Inventory-II (BDI-II; Beck et al., 1996) (in order to be included in the experiment, the score had to be less than 9, except in the depressed group, in which case the score had to be over 18); and the Young Mania Rating Scale (YMRS; Young et al., 1978) (depressed and euthymic patients could not have a score greater than 6 , while manic patients' scores had to be over 20). In addition, every participant completed the following questionnaires: (1) the Beck Anxiety Inventory (BAI; Beck et al., 1988), used as a measure of anxiety; and (2) the Social Adaptation Self-evaluation Scale (SASS; Bosc et al., 1997) to measure social functioning. Twenty-three patients and eight healthy controls were excluded according to the exclusion criteria. The demographic and clinical data for the final sample are presented in Table 1.

\subsection{The eye-tracking paradigm}

The stimuli included 80 images selected from the International Affective Picture System (IAPS; Lang et al., 2005). These stimuli were the same as those used by Kellough et al. (2008), and the images were categorized as happy, neutral, sad, or threatening. It is important to note that IAPS stimuli are rated in emotional parameters on a 9-point scale for valence (unpleasant $=1$, neutral $=5$, pleasant $=9$ ) and arousal $($ calm $=1$, excited $=9$ ), but not for specific emotional valence. For that reason, Kellough et al. (2008) conducted a pilot study to identify which valence (threatening or sad) best described the unpleasant pictures. A total of 20 trials (12 study +8 filler) were presented. Each trial began with a $1000 \mathrm{~ms}$ centrally presented fixation cross, followed by the simultaneous presentation of four images for $20 \mathrm{~s}$. Twelve study trials contained four images on each slide, one of 12 images selected from each of the following four valences: happy, neutral, sad, and threatening. For each trial, the localization of each image was randomly selected, with the constraint that each valence must occur in each of the four positions three times across the 12 trials. The presentation order of the images was randomized across the participants. Eight filler trials with four neutral images were presented to obscure the nature of the task.

\subsection{Apparatus}

A remote eye-tracking system (SMI RED250) was employed to measure the participants' eye movements, which allowed the participants free range of head movements. The sampling rate of the eye positions was $250 \mathrm{~Hz}$.
Table 1

Demographic and clinical data from control group, depressed, euthymic and manic patients. Data shown are averages and standard deviations.

\begin{tabular}{llllll}
\hline & $\begin{array}{l}\text { Control } \\
(N=20)\end{array}$ & $\begin{array}{l}\text { Euthymic } \\
(N=23)\end{array}$ & $\begin{array}{l}\text { Depressed } \\
(N=20)\end{array}$ & $\begin{array}{l}\text { Manic } \\
(N=23)\end{array}$ & $p$ \\
\hline \% Female & 50.0 & 34.8 & 45 & 39.1 & 0.76 \\
Age & $40.6(13.4)$ & $40.7(10.7)$ & $51.3(10.2)$ & $42.4(12.1)$ & 0.01 \\
SASS & $43.8(6.0)$ & $40.1(5.3)$ & $40.8(6.8)$ & $39.5(6.2)$ & 0.07 \\
\# of episodes & - & $6.2(5.7)$ & $6.8(3.1)$ & $7.2(6.1)$ & 0.79 \\
BAI & $10.0(5.1)$ & $5.6(4.1)$ & $24.0(8.7)$ & $11.2(7.8)$ & 0.00 \\
BDI & $4.8(3.2)$ & $2.9(3.4)$ & $27.0(7.2)$ & $5.1(3.7)$ & 0.00 \\
YMRS & - & $0.8(1.8)$ & $1.7(2.5)$ & $23.7(5.2)$ & 0.00 \\
Medication (\% of patients) & & & & \\
$\quad$ Lithium (\%) & - & 87.0 & 60.0 & 73.9 & 0.13 \\
Antiepileptic (\%) & - & 39.1 & 65.0 & 39.1 & 0.15 \\
Antipsychotic (\%) & - & 39.1 & 50.0 & 100.0 & 0.00 \\
Antidepressive (\%) & - & 0.0 & 60.0 & 0.0 & 0.00 \\
Anxiolytic (\%) & - & 30.4 & 90.0 & 91.3 & 0.00 \\
\hline
\end{tabular}

Note: The $p$ values correspond to the omnibus test for all groups.

\subsection{Procedure}

After signing an informed consent form, all participants completed a demographic interview, as well as the SSAS, BAI, and BDI-II rating scales. Additionally, patients participated in a clinical interview and responded to the YMRS. In a second session, which took place the following day, the participants completed the experiment. They were tested individually in a silent room, seated approximately $60 \mathrm{~cm}$ in front of the monitor in a height-adjustable chair. The experimental session began once the eye-tracker was successfully calibrated (i.e., average error was less than $1.5^{\circ}$ of the visual angle for each calibration point). The experimenter was located in the room and monitored the stimulus presentation and eye tracking throughout each trial. To promote lifelike information processing, participants were instructed via the computer screen to look freely at the images "as if you were watching television or looking at a photo album." The size of the pictures was $11.95^{\circ}$ (wide) $\times 9.18^{\circ}$ (high), and the distance between the pictures was $4.76^{\circ}$ (horizontal axis) and $3.53^{\circ}$ (vertical axis).

\subsection{Data analyses}

Data were computed using a velocity-based algorithm with a minimum fixation duration threshold of $100 \mathrm{~ms}$ and a peak velocity threshold of $40 \% \mathrm{~s}$. The areas of interest (AoIs) were also identified for each trial, and corresponded to the total area for each of the four images. To assess whether bipolar groups attended differentially to each valence over time, we computed (i) the percentage of fixation time attending to each image within each $5 \mathrm{~s}$ segment; (ii) the percentage of fixations (i. e., percentage of times that each subject fixated [and re-fixated] on a particular valence); (iii) the mean glance duration (i.e., the average time that each participant's gaze stayed within the boundaries of a particular valence, that is, a period beginning when the gaze entered the image, and ending when the gaze left the image); and (iv) the location of the first fixation (i.e., percentage of times that the first fixation lands in images of a particular valence).

\section{Results}

Although the hypotheses outlined in Section 1 led to specifically planned comparisons, for the sake of clarity, we conducted an omnibus analysis of variance (ANOVA) for each dependent measure. The percent fixation, mean glance duration, and percent location of the first fixation were examined in separate ANOVAs with Group (control, euthymic, depressed, manic) as a betweensubject factor and Valence (neutral, happy, sad, threatening) as a within-subject factor. The analyses of the percent fixation time also included Time Segment $(1-5,5-10,10-15,15-20 \mathrm{~s})$ as a within-subject factor. The descriptive data for each condition are presented in Table 2. When the effects were significant, we conducted the following series of tests to control for type I errors: (i) Dennett's test was used to compare each group of BD relative to the appropriate control group (i.e., between-group comparisons; 
Table 2

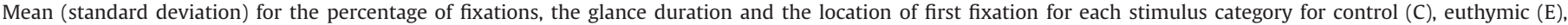
depressed (D) and manic (M) groups.

\begin{tabular}{|c|c|c|c|c|c|c|c|c|c|c|c|c|}
\hline \multirow[t]{2}{*}{ Stimulus category } & \multicolumn{4}{|c|}{ Number of fixations (\% Of total) } & \multicolumn{4}{|c|}{ Glance duration (ms) } & \multicolumn{4}{|c|}{ Location of first fixation (\% of total) } \\
\hline & $\mathrm{C}$ & E & $\mathrm{D}$ & M & $\mathrm{C}$ & $\mathrm{E}$ & $\mathrm{D}$ & M & $\mathrm{C}$ & $\mathrm{E}$ & $\mathrm{D}$ & M \\
\hline Neutral & $18.5(5.6)$ & $20.8(4.1)$ & $19.8(5.2)$ & $18.4(6.2)$ & $427(244)$ & $532(248)$ & $454(277)$ & $370(238)$ & $17.5(11.8)$ & $19.9(6.0)$ & $20.4(6.3)$ & $20.1(14.0)$ \\
\hline Happy & $38.3(13.3)$ & $31.7(6.7)$ & $26.8(6.3)$ & $33.4(14.5)$ & 939 (679) & $848(452)$ & $643(426)$ & $492(451)$ & $34.2(13.5)$ & $32.2(8.4)$ & $30.0(9.1)$ & $32.1(16.4)$ \\
\hline Sad & $25.2(7.6)$ & $23.8(4.0)$ & $27.2(6.2)$ & $23.3(8.4)$ & $627(380)$ & $607(267)$ & $626(390)$ & $467(267)$ & $23.3(7.9)$ & $19.6(9.3)$ & $23.3(11.0)$ & $24.1(11.7)$ \\
\hline Threat & $18.0(6.7)$ & $23.7(7.4)$ & $26.2(6.7)$ & $24.9(11.5)$ & $430(293)$ & $589(282)$ & $609(321)$ & $480(278)$ & $25.0(11.5)$ & $28.2(10.0)$ & $26.2(9.8)$ & $23.7(13.8)$ \\
\hline
\end{tabular}

see Miller, 1981); and (ii) Bonferroni post hoc tests were used to analyze the effect of Valence (i.e., within-group comparisons).

\subsection{Percent fixation time}

The ANOVA of the percent fixation time revealed an effect of Time Segment, $F(3,246)=15.53, p<0.001, \eta^{2}=0.16$, which was qualified by a Valence $\times$ Time Segment interaction, $F(9,738)=4.59$, $p<0.001, \eta^{2}=0.05$. The ANOVA also revealed an effect of Valence, $F(3,246)=28.32, p<0.001, \eta^{2}=0.26$, which was qualified by a Valence $\times$ Group interaction, $F(9,246)=3.09, p=0.002, \eta^{2}=0.10$. The other interactions were not significant (all $F$ values $<1$ ). ${ }^{1}$

Simple test effects on the effect of the group for each valence were conducted to examine the Valence $\times$ Group interaction (Fig. 1). These analyses revealed that there were differences between the groups for the happy images, $F(3,82)=3.56$, $p=0.018, \eta^{2}=0.12$. Dunnett's test showed that patients in a depressive state spent less time viewing happy images than healthy individuals $(p=0.002)$. We also found differences between the groups for threatening images, $F(3,82)=4.08, p=0.009$, $\eta^{2}=0.13$, and Dunnett's test revealed that patients in euthymic, depressive, and manic states spent more time attending to threatening stimuli than healthy individuals $(p=0.026, p=0.002$, $p=0.012$, respectively). In contrast, there were no significant between-group differences for the sad or neutral images, $F$ $(3,82)=1.43, p=0.24$, and $F<1$, respectively.

To examine the Valence $\times$ Time Segment interaction, trend analyses concerning the effect of time for each valence were conducted (Fig. 2). For the positive stimuli, the percent fixation time increased linearly over $20 \mathrm{~s}, F(1,85)=11.38, p=0.001$, $\eta^{2}=0.12$. For the neutral and sad stimuli, the effect of the Time Segment was not significant, at $F(3,246)=1.57, p=0.20$ and $F$ $(3,246)=1.03, p=0.38$, respectively (i.e., percent fixation time was relatively stable over the $20 \mathrm{~s}$ period). Finally, for threatening stimuli, there were significant linear, $F(1,85)=17.43, p<0.001$, $\eta^{2}=0.17$, and quadratic, $F(1,85)=9.18, p=0.003, \eta^{2}=0.10$, effects of Time Segment, and the percent fixation time initially decreased over time, but then increased toward the end of the trial. This pattern of data was similar for the patients and healthy controls, as deduced by the lack of a significant three-way interaction, $F<1$.

\subsection{Percent fixations}

The ANOVA of the percent fixations revealed an effect of Valence, $F(3,246)=29.68, p<0.001, \eta^{2}=0.27$, and a significant

\footnotetext{
${ }^{1}$ According to Table 1, there were significant differences between the groups in the age and BAI scores. Analyses of covariance (ANCOVAs) were completed that included age and BAI score as covariates. Neither age nor anxiety symptoms significantly interacted with any of the main effects or interactions in the previous analysis ( $p>0.33$ and $p>0.14$, respectively). Furthermore, despite the strong BAIBDI correlation $(r=0.72)$, the interaction effect was very similar to that in analyses that did not include BAI as a covariate. Thus, anxiety symptoms were not the main cause of the differences across the groups.
}

Valence $\times$ Group interaction, $F(9,246)=3.08, p=0.002, \eta^{2}=0.10$. Simple effect tests on the effects of the groups for each valence were conducted to examine the Valence $\times$ Group interaction. These analyses revealed that there were differences between the groups for the happy images, $F(3,82)=3.82, p=0.013, \eta^{2}=0.12$. Dunnett's test indicated that the percent of fixations on the happy stimuli was lower in the individuals in the depressed group than in the control group $(p=0.002)$. The analyses for threatening images also showed that there were differences among the groups, $F(3,82)=3.77, p=0.014, \eta^{2}=0.12$. Dunnett's test revealed that the percent of fixations on the threatening stimuli was higher for the euthymic, depressed, and manic groups, than for the healthy controls ( $p=0.038, p=0.004, p=0.011$, respectively). There were no significant differences across the groups for the percent of fixations on the sad images, $F(3,82)=1.38, p=0.26$, or on the neutral images, $F(3,82)=1.01, p=0.39$.

\subsection{Mean glance duration}

The ANOVA of the mean glance duration revealed an effect of Valence, $F(3,246)=23.71, p<0.001, \eta^{2}=0.22$, which was qualified by a Valence $\times$ Group interaction, $F(9,246)=2.05, p=0.035$, $\eta^{2}=0.07$. The effect for the Group was not significant, $F(3,82)=$ $1.11, p=0.40$. Simple effect tests on the effects of the groups for each valence were conducted to examine the Valence $\times$ Group interaction. However, these analyses did not reveal any significant difference between the groups for the happy images, $F(3,82)=1.51$, $p=0.22$, sad images, $F(3,82)=1.23, p=0.30$, threatening images, $F(3,82)=1.80, p=0.15, \eta^{2}=0.06$, or neutral images, $F(3,82)=1.64$, $p=0.19$.

\subsection{Location of the initial fixation}

The ANOVA of the location of the first fixation revealed an effect of Valence, $F(3,246)=15.33, p<0.001, \eta^{2}=0.16$. The Valence $\times$ Group interaction did not approach significance, $F<1$. Bonferroni comparisons revealed that the participants' first fixations were directed more toward happy images than toward the other types of images (all $p<0.034$ ).

\section{Discussion}

To the best of our knowledge, this is the first eye-tracking experiment that has examined attentional biases during the processing of (multiple) emotional images in patients with BD (in manic, depressive, and euthymic states). The main findings of this experiment can be summarized as follows. First, the attentional biases toward mood-relevant images (i.e., happy or sad stimuli) in BD patients were dependent on their clinical episodes. This was particularly clear concerning happy images in individuals in a depressive state. Second, the attentional biases toward threatening images in bipolar patients occurred regardless of their episode. Third, these attentional biases (i.e., the avoidance of 


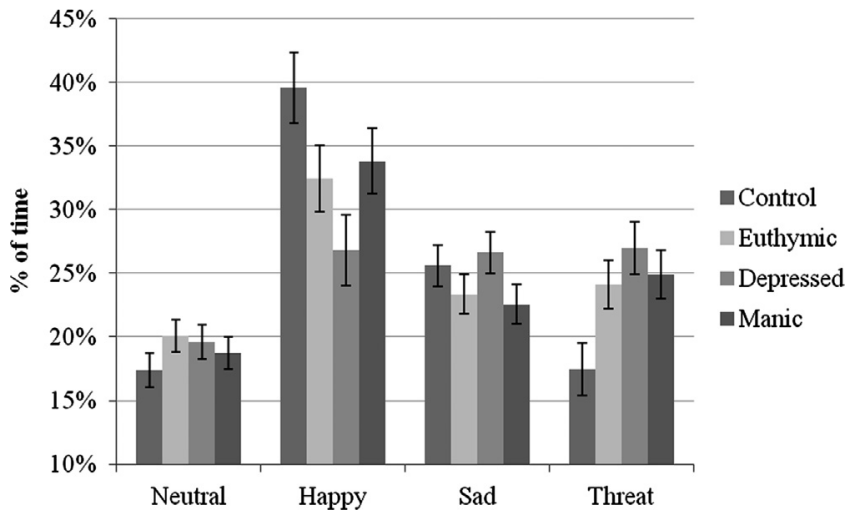

Fig. 1. Percent time attending each category for control, euthymic, depressed, and manic groups.

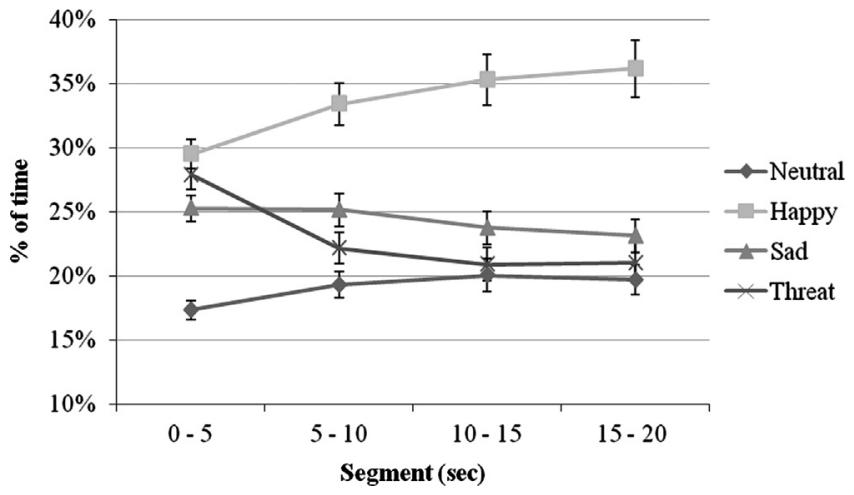

Fig. 2. Percent time attending each category across 5-s time segments.

happy stimuli by depressed patients and the preference for threatening stimuli by all groups of BD patients relative to healthy individuals) remained stable across a period of $20 \mathrm{~s}$ (i.e., the presentation time of stimuli did not condition the valence-group interaction). Finally, the initial fixation was not affected by mood; it was only influenced by image valence (i.e., there were more initial fixations for happy and threatening images). We will now discuss how these findings help to shed light on the processing of emotional images by bipolar patients.

Regarding the nature of emotional biases in individuals with $\mathrm{BD}$, the biases toward happy and sad images were statedependent. On the one hand, similarly to MDD patients (Kellough et al., 2008; Leyman et al., 2011; Sears et al., 2010, 2011; Ellis et al., 2011), BD patients in the depressive phase paid less attention to the happy images than the control group, as deduced from the fixation time and the fixation frequency found for these images. On the other hand, although BD patients in the depressive phase paid more attention to sad images than the healthy controls in percent of fixations (27\% versus $25 \%$, respectively), this difference did not reach statistical significance-this difference was significant in previous eye-tracking studies in individuals with MDD (Eizenman et al., 2003; Kellough et al., 2008). Thus, an "anhedonic bias" was the most salient disturbance in bipolar depression. This emotional bias in BD patients may be easily explained by a lack of motivation to focus their attention on positive stimuli than by a higher attention toward sad images. Taken together, this altered scanning of emotionally relevant information in patients experiencing depressive episodes is in part consistent with recent behavioral evidence (García-Blanco et al., 2013) and with the theoretical models of BD functioning (Beck, 1976). It may be important to note that BD patients in the manic group did not show an abnormal sensitivity to happy or sad images relative to the control group; there was only a nonsignificant trend in which these patients paid less attention to the sad images than the control group in terms of percent fixations ( $23 \%$ versus $25 \%$, respectively). The manic patients did not show any clear trend, at least during the extended viewing of competing stimuli in the present experiment. This pattern could reflect conflicting positive and negative appraisals in mania (Mansell et al., 2007). In summary, the avoidance of positive stimuli in the depressive state may disturb emotional self-regulation and may be an important maintenance factor for BD.

A remarkable finding in the present eye-tracking experiment is that the biased processing of threatening information may constitute a general vulnerability factor of $\mathrm{BD}$, as revealed by the comparison in percent fixation time and percent fixations on threatening images of all patient groups relative to the healthy individuals. Cognitive vulnerability-stress theories (Beck, 1976) suggest that the presence of dysfunctional cognitive schemata in BD patients conditions their attentional preferences even during asymptomatic states, which constitutes a potential mechanism of vulnerability when euthymic patients experience stressful life events. In these circumstances, the attentional preference toward threatening stimuli would increase the BD patients' emotional reactivity and would contribute to the onset or exacerbation of an affective episode. This bias toward threatening information is in accordance with accounts that emphasize the influence of these pathogenic attentional biases on reactivity to stressful events for BD patients, even in asymptomatic patients and high-risk relatives (e.g., see Gotlib et al., 2005; Alloy et al., 2006). In this respect, Gotlib et al. (2005) reported that, after the induction of a negative mood, the offspring of parents with BD exhibited an attentional bias toward threatening and manic-irritable information, but not toward positive or negative information. Furthermore, as indicated in Section 1, fMRI studies have shown a disturbance in neural activation during fear or threat processing across the different states of BD (including asymptomatic patients) using a variety of emotional paradigms (see Chen et al., 2006, for patients with mania and bipolar depression; see Wessa et al., 2007, for an example with euthymic patients). It is important to keep in mind that, unlike what occurs in $\mathrm{BD}$, this threat related attentional bias is absent in unipolar depression (see Armstrong and Olatunji, 2012). The long fixation times for threatening images would reflect a difficulty to disengage attention from those pictures, and this may contribute to the rumination on threatening information by its through continuous processing-note that MDD has been related with rumination process about sad topics instead (Koster et al., 2011). This pattern supports recent theoretical proposals emphasizing the distinctive traits related to BD and MDD (Mansell et al., 2007).

Regarding the temporal course of emotional processing, there were no group differences in the localization of the first fixation on the emotional images. Thus, we found no signs of differential attentional biases in the patients versus controls during the initial orientation (i.e., in the earliest stages of information processing). Most participants directed their first fixations toward positive images; this pattern is also typical of healthy individuals (Calvo et al., 2007). Similarly to Armstrong and Olatunji's (2012) metaanalysis with MDD individuals, the differences in the attentional biases in the patients relative to the healthy controls were robust in the measures computed for relatively long exposure durations (i.e., $5 \mathrm{~s}$; that is, during later stages in information processing, as reflected by fixation time [which indicates sustained attention at encoding] and fixation frequency [which indicates re-orienting of attention]). The decrease in attention toward happy images among depressed patients versus healthy controls and the increase in attention toward threatening images by all BD patients versus healthy controls did not change across time segments. 
The present study comes with certain limitations that are typical in studies with patients. All patients in this study were taking psychotropic medication (see Table 1). In order to examine the role of medication on the obtained effects, we performed post hoc ANCOVAs on the relationships between the magnitude of the mood effect and medication; however, there were no signs of an effect (all $F$ values $<1$ ). Although we acknowledge that a comparison group of patients with MDD would have been relevant when it came to examining the differences between emotional processing in unipolar and bipolar depression, the main focus of the experiment was an examination of the intricacies of attentional biases to emotional images in the different episodes of BD rather than a comparison between these mental disorders. In addition, while the use of complex images may increase realism and generalizability, it may also reduce experimental control. As one reviewer pointed out, the simultaneous presentation of multiple stimuli has the added benefit of allowing investigation of whether the presence of evocative stimuli does not disrupt biased attention for mood-relevant information; however, this may also have reduced the independent effects of the emotional stimuli.

In conclusion, the present experiment revealed that $\mathrm{BD}$ patients experiencing depressive episodes showed differential attention processing for happy images, suggesting an anhedonic lack of sensitivity to positive stimuli. In addition, we found a general preference for threatening images in BD patients, which occurred even in asymptomatic patients. We believe that this state/trait dissociation allows for a better characterization of this episodic and chronic mental disorder. From a clinical perspective, it will be important to determine the following in future research (i) whether the training of the biases toward mood-relevant information may constitute a novel treatment for symptomatic BD episodes (see Wadlinger and Isaacowitz, 2008, for evidence in patients with MDD) and (ii) whether attentional biases toward threatening information constitute a marker of vulnerability for $\mathrm{BD}$, and if so, what preventive strategies may be employed, particularly in high-risk relatives.

\section{Acknowledgements}

This research has been partly supported by grants PSY201126924 and EDU2011-25885 from the Spanish Ministry of Economy and Competitiveness. Ana García-Blanco was the recipient of a postgraduate grant from the program "Atracció de Talent" at the University of Valencia (VLC-Campus).

\section{References}

Alloy, L.B., Abramson, L.Y., Walshaw, P.D., Neeren, A.M., 2006. Cognitive vulnerability to unipolar and bipolar mood disorders. Journal of Social and Clinical Psychology 25, 726-754, http://dx.doi.org/10.1521/jscp.2006.25.7.726.

American Psychiatric Association, 2000. Diagnostic and Statistical Manual of Mental Disorders (Text Revision). Author, Washington, DC

Armstrong, T., Olatunji, B.O., 2012. Eye tracking of attention in the affective disorders: a meta-analytic review and synthesis. Clinical Psychology Review 32, 704-723, http://dx.doi.org/10.1016/j.cpr.2012.09.004.

Beck, A.T., 1976. Cognitive Therapy and the Emotional Disorders. New American Library, New York

Beck, A.T., Epstein, N., Brown, G., Steer, R.A., 1988. An inventory for measuring clinical anxiety: psychometric properties. Journal of Consulting and Clinical Psychology 56, 893-897, http://dx.doi.org/10.1037//0022-006X.56.6.893.
Beck, A.T., Steer, R.A., Brown, G.K., 1996. Manual for the Beck Depression Inventory-II. Psychological Corporation, San Antonio, TX

Bosc, M., Dubini, A., Polin, V., 1997. Development and validation of a social functioning scale, the Social Adaptation Self-Evaluation Scale. European Neuropsychopharmacology 7, S57-S70, http://dx.doi.org/10.1016/S0924-977X(97) 00420-3.

Calvo, M.G., Nummenmaa, L., Hyönä, J., 2007. Emotional and neutral scenes in competition: orienting, efficiency, and identification. Quarterly Journal of Experimental Psychology 60, 1585-1593, http://dx.doi.org/10.1080/ 17470210701515868.

Chen, C.-H., Lennox, B., Jacob, R., Calder, A., Lupson, V., Bisbrown-Chippendale, R., Suckling, J., Bullmore, E., 2006. Explicit and implicit facial affect recognition in manic and depressed states of bipolar disorder: a functional magnetic resonance imaging study. Biological Psychiatry 59, 31-39, http://dx.doi.org/10.1016/ j.biopsych.2005.06.008.

Eizenman, M., Yu, L., Grupp, L., Eizenman, E., Ellenbogen, M., Germar, M., Levitan, R.D. 2003. A naturalistic visual scanning approach to assess selective attention in major depressive disorder. Psychiatry Research 118, 117-128, http://dx.doi.org/ 10.1016/S0165-1781(03)00068-4.

Ellis, A.J., Beevers, C.G., Wells, T.T., 2011. Attention allocation and incidental recognition of emotional information in dysphoria. Cognitive Therapy and Research 40, 443-454, http://dx.doi.org/10.1007/s10608-010-9305-3.

García-Blanco, A.C., Perea, M., Livianos, L., 2013. Mood-congruent bias and attention shifts in the different episodes of bipolar disorder. Cognition and Emotion 27, 1114-1121, http://dx.doi.org/10.1080/02699931.2013.764281.

Gotlib, I.H., Traill, S.K., Montoya, R.L., Joormann, J., Chang, K., 2005. Attention and memory biases in offspring of parents with bipolar disorder: implications from a pilot study. Journal of Child Psychology and Psychiatry 46, 84-93, http://dx. doi.org/10.1111/j.1469-7610.2004.00333.x.

Hermans, D., Vansteenwegen, D., Eelen, P., 1999. Eye movement registration as a continuous index of attention deployment: data from a group of spider anxious students. Cognition and Emotion 13, 419-434, http://dx.doi.org/10.1080/ 026999399379249.

Jongen, E.M., Smulders, F.T., Ranson, S.M., Arts, B.M., Krabbendam, L., 2007. Attentional bias and general orienting processes in bipolar disorder. Journal of Behavior Therapy and Experimental Psychiatry 38, 168-183, http://dx.doi. $\operatorname{org} / 10.1016 / j . j b t e p .2006 .10 .007$.

Kellough, J., Beevers, C.G., Ellis, A., Wells, T.T., 2008. Time course of selective attention in depressed young adults: an eye tracking study. Behavior Research and Therapy 11, 1238-1243, http://dx.doi.org/10.1016/j.brat.2008.07.004.

Koster, E.H., De Lissnyder, E., Derakshan, N., De Raedt, R., 2011. Understanding depressive rumination from a cognitive science perspective: the impaired disengagement hypothesis. Clinical Psychology Review 31, 138-145.

Lang, P.J., Bradley, M.M., Cuthbert, B.N., 2005. International Affective Picture System (IAPS): Affective Ratings of Pictures and Instruction Manual (Technical report A-6). University of Florida, Gainesville, FL

Leyman, L., De Raedt, R., Vaeyens, R., Philippaerts, R., 2011. Attention for emotional facial expressions in dysphoria: an eye movement registration study. Cognition and Emotion 25, 111-120, http://dx.doi.org/10.1080/02699931003593827.

Mansell, W., Morrison, A.P., Reid, G., Lowens, I., Tai, S., 2007. The interpretation of, and responses to, changes in internal states: an integrative cognitive model of mood swings and bipolar disorders. Behavioural and Cognitive Psychotherapy 35, 515-539, http://dx.doi.org/10.1017/S1352465807003827.

Miller, R., 1981. Simultaneous Statistical Inference, 2nd ed. Springer-Verlag, New York

Rayner, K., 2009. Eye movements and attention in reading, scene perception, and visual search. Quarterly Journal of Experimental Psychology 62, 1457-1506, http://dx.doi.org/10.1080/17470210902816461.

Sears, C.R., Newman, K.R., Ference, J.D., Thomas, C.L., 2011. Attention to emotional images in previously depressed individuals: an eye-tracking study. Cognitive Therapy and Research 35, 517-528, http://dx.doi.org/10.1007/s10608-0119396-5.

Sears, C.R., Thomas, C.L., LeHuquet, J.M., Johnson, J.C.S., 2010. Attentional biases in dysphoria: an eye tracking study of the allocation and disengagement of attention. Cognition and Emotion 24, 1349-1368, http://dx.doi.org/10.1080/ 02699930903399319.

Wadlinger, H.A., Isaacowitz, D.M., 2008. Looking happy: the experimental manipulation of a positive visual attention bias. Emotion 8, 121-126, http://dx.doi. org/10.1037/1528-3542.8.1.121.

Wessa, M., Houenou, J., Paillère-Martinot, M.L., Berthoz, S., Artiges, E., Leboyer, M., Martinot, J.L., 2007. Fronto-striatal overactivation in euthymic bipolar patients during an emotional go/no-go task. American Journal of Psychiatry 164, 38-46, http://dx.doi.org/10.1176/appi.ajp.164.4.638.

Young, R.C., Biggs, J.T., Ziegler, V.E., Meyer, D.A., 1978. A rating scale for mania: reliability, validity and sensitivity. British Journal Psychiatry 133, 429-435, http://dx.doi.org/10.1192/bjp.133.5.429. 